\title{
Nami Azar and Carolyn K. Donaldson (Eds): RadCases. Ultrasound Imaging. Thieme Publishers New York 2015, ISBN: 978-1-60406322-6
}

\author{
Vincenzo Parlato ${ }^{1}$
}

Published online: 2 April 2016

(C) Springer-Verlag Berlin Heidelberg 2016

RadCases - Ultrasound Images is a publication that is part of a series describing radiological cases, directed by Jonathan Lorenz and Hector Ferral for Thieme Publishers. The editors of this volume are Nami Azar, Associate Professor of Radiology at the University Hospital Case Medical Center in Cleveland, and Carolyn K. Donaldson, Clinical Assistant Professor at the University of Chicago.

The book concerns ultrasound imaging, and it proves to be very useful both for residents and for expert users in that it is structured using very detailed ultrasonographic images, extensively explained with plain and didactic language. As is the cases for all the other titles of the series, the publication includes a scratch-off code that allows 12 months of access to a searchable online database of all 100 patients from the book plus an additional 150 cases, therefore reaching a total of 250 patients.

Most of the cases, concerning a wide variety of pathologies, are correlated with other imaging techniques such as computed tomography (CT) and magnetic resonance imaging (MRI). The text includes a vast number of images, referring either to the most frequent occurrences in clinical practice, or to some more complex radiological problems. A particular quality of such a book is in the fact that the authors do not only describe the pathology, which is in evidence by exclusively observing the image. Rather, for each case, they underline a series of aspects that may have relevance in that pathological condition, such as sex, age, symptoms, and past and present clinical history. In this way, the final diagnosis may be reached not only by evaluating the image, but also through the complementary information, which can sometimes be insightful in setting out the goal, unreachable by diagnostic imaging alone. Furthermore, the authors set forth, for every case, some particulars contained in the image which could mislead even the most expert operator as pitfalls, and others which instead are without any doubt a primary support to carrying out the final diagnosis as pearls. In this way, a decidedly ample panorama of core elements able to help in formulating a correct diagnosis is offered to the readers.

The method chosen in this book to expose the ultrasonographic problems that are dealt with stimulates in the readers a particular attention. Thanks to the vast amount of given indications and of aspects that are taken into consideration, everyone who benefits from this perusal does not feel like a simple reader, but is personally involved in the solution of each clinical case.

Thus, this publication - and this is the greatest merit for which I recognize the authors - is not exclusively a theoretical text to be studied, but also a practical book of exercises, helpful both for the beginner and for the expert ultrasonographer, who may be aided in his/her daily practice.

Vincenzo Parlato

VINCENZO.PARLATO@unina2.it

1 Second University of Naples, Napoli, Italy 